\title{
Optimization of Chemical-Technological Processes in the Face of Uncertainty
}

\author{
V. A. Pogonin \\ Department of Information Processes and Management, Tambov State Technical University, Sovetskaya St., 106, Tambov, Russia
}

Received 20 July 2020; Accepted 20 October 2020

\begin{abstract}
A wide class of chemical - technological processes is characterized by a random nature of the change in parameters that affect the change in the characteristics of control objects. The characteristics of the process itself are random, which include physicochemical constants, heat and mass transfer coefficients, rates of chemical reactions, concentration of substances in input flows, etc. The development of control systems in conditions of uncertainty of the parameters of chemical-technological processes with guaranteed provision for the fulfillment of all restrictions is a significant problem. Existing approaches can lead to inaccurate solutions and increased computation time and do not guarantee the fulfillment of technological and technical constraints. Development of a method for solving problems of optimization of chemical technological processes in conditions of uncertainty with a guarantee of the fulfillment of technological limitations. A method for solving problems of guaranteed optimization of chemical-technological processes in conditions of uncertainty was proposed, which allows to significantly reduce the time for solving such problems. Algorithms for the synthesis of an auxiliary mathematical model and the choice of a method for solving the problem of ensuring optimization under uncertainty, formulation and results of solving the problem of guaranteed optimization of the process of obtaining Scheffer's acid in a microwave reactor under uncertainty are presented.
\end{abstract}

Keywords: chemical-technological process, optimization, uncertainty

\section{Introduction}

Development chemical productions characterized by tightening requirements for finished product quality and environmental cleanliness, introduction of technologies providing a high level of energy and resource conservation. This leads to the fact that errors in the management of chemicaltechnological processes (CTP) can lead to huge economic losses and contribute to emergency situations. Therefore, there is a need to consider management tasks, the solution of which would ensure the implementation of technological and technical requirements $\varphi_{i}$ with a given guarantee $a_{i}, i=\overline{1, n}$ ( $n$-number of technological requirements) $[1,2]$.

Obviously, the information received directly from the control object is often inaccurate (uncertain), which is caused not only by errors in the technical means of measuring and transmitting information, but also by the stochasticity of the objects themselves or of the transmission channels material and energy impacts [3,4] Parameters related to CTP can also be undefined (thermophysical characteristics of materials, heat and mass transfer coefficients, concentration of impurities in raw materials, etc.) $[4,5]$.

Existing theoretical principles and methods for solving the problems of guaranteeing optimization and control of CTP using the apparatus of mathematical statistics and interval analysis to formalize uncertainties cannot be applied to most modern chemical productions. Moreover, it is impossible to use these methods for newly designed productions. Thus, well-known approaches can lead to inaccurate

*E-mail address: pogvas@inbox.ru

ISSN: 1791-2377 @ 2020 School of Science, IHU. All rights reserved. doi:10.25103/jestr.136.21 solutions, increase the computation time and do not guarantee the implementation of technological and technical limitations $[6,7]$.

Existing approaches can lead to inaccurate solutions and increase the computation time and do not guarantee the implementation of technological and technical limitations [7$10]$.

It is obvious that under these conditions it is reasonable to use the mathematical apparatus of the theory of fuzzy sets, which allows one to formalize the uncertainty of the parameters of chemical-technological processes, using the accumulated knowledge and expert estimates.

The aim of the research is development of a method for solving problems of optimization of chemical technological processes in conditions of uncertainty with a guarantee of the fulfillment of technological limitations and verification of its effectiveness in the management of technological process.

\section{Main part}

We formulate the problem of guaranteeing optimization of CTP under uncertainty presented in [1] in the following simplified form: it is necessary to find a control vector $\mathbf{u}^{*}$, such that the objective function $Q(\mathbf{u})$ takes a minimum value

$\mathbf{u}^{*}=\arg \min _{u \in D} Q(u)$

where $D=\left\{\mathbf{u} \mid \mathbf{u} \in \mathrm{U} \wedge \varphi_{\mathrm{i}}^{\mathrm{b}}(\mathbf{u}) \geq \mathrm{a}_{\mathrm{i}}, \quad i=\overline{1, n}\right\}$ - area admissible management, 
$\varphi_{i}^{\mathrm{b}}(\mathbf{u})=\min _{E_{i}} \varphi_{\mathrm{i}}$

$E_{i}=\left\{\varphi_{i} \mid \mu_{\tilde{\mathfrak{J}}}^{\mathrm{i}}\left(\varphi_{i} \mid \mathbf{u}\right) \geq \varepsilon_{i}\right\}$ - significance area a bunch of $\varphi_{\mathrm{i}}$,

$\varepsilon_{i}$ - constant magnitude "significance level",

$\mu_{\tilde{\mathfrak{I}}}^{\mathrm{i}}\left(\varphi_{\mathrm{i}} \mid \mathbf{u}\right)=\max _{x, y} \min \left(\mu_{\tilde{X}}(\mathbf{x}), \mu_{\tilde{Y}}(\mathbf{y} \mid \mathbf{u})\right)$

$\mid \varphi_{i}=\varphi_{\mathrm{i}}(\mathbf{x}, \mathbf{y}, \mathbf{u})$

$\mu_{\widetilde{Y}}(\mathbf{y} \mid \mathbf{u})=\max _{x, b} \min \left(\mu_{\tilde{X}}(\mathbf{x}), \mu_{\tilde{B}}(\mathbf{b})\right)$

$\mid \mathbf{y}=M(\mathbf{x}, \mathbf{u}, \mathbf{b})$.

The solution of the guaranteeing optimization problem is associated with significant difficulties in multiple calculations, both of the equations of the mathematical model $\mathrm{M}$ and of the constraint systems due to the need to calculate the membership functions of the output quantities and process parameters by famous membership function input quantities.

Consider the optimization method using a two-model complex (two-model optimization), which is a development of the theory of $\alpha$-problems [2].

The idea of two-model optimization is reduced to replacing the $\alpha$-problem with some "auxiliary" optimization problem.

Will accept the following notation: denote the "auxiliary" mathematical model by the operator $\mathbf{m}$

$\mathbf{y}=\mathbf{m}(\mathbf{x}, \mathbf{u})$

We introduce the "auxiliary" objective function $q(\mathbf{u}, \mathbf{y})$ and the system of operators $\mathrm{f}_{\mathrm{i}}(\mathbf{y}, \mathbf{u})$

$$
f_{i}: Y \times U \rightarrow R
$$

Given the notation, we formulate task the two-model optimization: it is necessary to find the vectors $\boldsymbol{\alpha}^{*}=\left(\alpha_{1}^{*}, \alpha_{2}{ }_{2}\right.$, $\left.\ldots, \alpha_{\mathrm{n}}^{*}\right)$ and $\mathbf{u}^{*}$, at which the objective function $\mathrm{q}^{*}(\boldsymbol{\alpha}) \quad \mathrm{q}$ $\left(\mathbf{u}_{\alpha}{ }^{\text {opt }}, \mathbf{y}_{\alpha}{ }^{\text {opt }}\right)$

$\boldsymbol{\alpha}^{*}=\arg \min _{\alpha} q\left(\mathbf{u}_{\alpha}{ }^{\text {opt }}, \mathbf{y}_{\alpha}{ }^{\text {opt }}\right)$

subject to the conditions

$\varphi_{\mathrm{i}}^{\mathrm{b}}\left(\mathbf{u}_{\alpha}^{*}\right) \geq \mathrm{a}_{\mathrm{i}}, \quad i=\overline{1, n}$,

where $\varphi_{\mathrm{i}}^{\mathrm{b}}\left(\mathbf{u}_{\alpha}{ }^{\mathrm{opt}}\right)=\min _{E_{i}} \varphi_{\mathrm{i}}$,

$E_{i}=\left\{\varphi_{\mathrm{i}} \mid \mu_{\tilde{\mathfrak{S}}}^{\mathrm{i}}\left(\varphi_{\mathrm{i}} \mid u_{\alpha}^{o p t}\right) \geq \varepsilon_{\mathrm{i}}\right\}$,

$\mu_{\widetilde{J}^{\mathrm{i}}}^{\mathrm{i}}\left(\varphi_{\mathrm{i}} \mid u_{\alpha}^{o p t}\right)=\max _{x, y} \min \left(\mu_{\tilde{X}}(\mathbf{x}), \mu_{\tilde{Y}}\left(\mathbf{y} \mid u_{\alpha}^{o p t}\right)\right)$

$\mid \varphi_{\mathrm{i}}=\varphi_{\mathrm{i}}\left(\mathbf{x}, \mathbf{y}, u_{\alpha}^{o p t}\right)$,

$\mu_{\tilde{Y}}\left(\mathbf{y} \mid \mathbf{u}_{\alpha}{ }^{\mathrm{opt}}\right)=\max _{x, b} \min \left(\mu_{\tilde{X}}(\mathbf{x}), \mu_{\tilde{B}}(\mathbf{b})\right)$

$\mid \mathbf{y}=\boldsymbol{M}(\mathbf{x}, \mathbf{u}, \mathbf{b})$

where $\mathbf{u}_{\alpha}{ }^{\text {opt }}$ determined algorithmically by solving the task $\mathbf{u}_{\alpha}{ }^{\text {opt }}=\arg \min _{u \in U_{\alpha}} q(\mathbf{y}, \mathbf{u})$,

where $\mathrm{U}_{\alpha}=\left\{\mathbf{u} \mid \mathrm{f}_{\mathrm{i}}(\mathbf{y}, \mathbf{u}) \geq \alpha_{\mathrm{i}}, \quad \mathrm{i}=\overline{1, \mathrm{n}}, \mathbf{y}=\mathbf{m}(\mathbf{x}, \mathbf{u})\right\}$. Wherein $\mathbf{u}^{*}=\mathbf{u}_{\alpha^{*}}{ }^{\text {opt }}$.

Two-model optimization can be used as a high-speed method for solving the task of guaranteeing optimization of CTP under conditions of uncertainty.

However, the use of two-model optimization is possible only with a certain correspondence between the operators $M$, $J, \varphi$ and the operators $\mathbf{m}, q, f$.

We formulate a theorem defining the identity conditions for a two-model optimization task and the tasks of guaranteeing optimization in the face of uncertainty.

Theorem. Let the task of ensuring optimization under uncertainty have a solution and let the models $\boldsymbol{M}(\mathbf{x}, \mathbf{u}, \mathbf{b}), \mathbf{m}$ $(\mathbf{x}, \mathbf{u})$ and functionals $Q(\mathbf{u}), \varphi_{\mathrm{i}}(\mathbf{x}, \mathbf{y}, \mathbf{u}), \mathrm{q}(\mathbf{u}, \mathbf{y}), f_{i}(\mathbf{y}, \mathbf{u})$ such, what for any $\mathbf{u}_{1}, \mathbf{u}_{2} \in U$ following relations are fulfilled:

$\left[q\left(\mathbf{u}_{1}, \mathbf{y}\right) \mid \mathbf{y}=\mathbf{m}\left(\mathbf{x}, \mathbf{u}_{1}\right)\right]>$

$>\left[q\left(\mathbf{u}_{2}, \mathbf{y}\right) \mid \mathbf{y}=\mathbf{m}\left(\mathbf{x}, \mathbf{u}_{2}\right)\right] \Rightarrow$

$\Rightarrow\left[Q\left(\mathbf{u}_{1}\right)=\left(\min \mathrm{J} \mid \mu_{\tilde{J}}\left(\mathrm{~J} \mid \mathbf{u}_{1}\right)>\mu_{\diamond}\right)\right]>$

$>\left[Q\left(\mathbf{u}_{2}\right)=\left(\min \mathrm{J} \mid \mu_{\tilde{J}}\left(\mathrm{~J} \mid \mathbf{u}_{2}\right)>\mu_{\star}\right)\right]$,

$f_{i}\left(\mathbf{y}, \mathbf{u}_{1}\right) \geq \mathrm{f}_{\mathrm{i}}\left(\mathbf{y}, \mathbf{u}_{2}\right) \Rightarrow \varphi_{\mathrm{i}}^{\mathrm{b}}\left(\mathbf{u}_{1}\right) \geq \varphi_{\mathrm{i}}^{\mathrm{b}}\left(\mathbf{u}_{2}\right)$,

then there exists an $\alpha$-task such that its solution coincides with the solution of the task of guaranteeing optimization under conditions of uncertainty.

The proof of the theorem due to cumbersomeness is not given.

The proved theorem, supplemented by a number of similar theorems, are used in the synthesis of the auxiliary mathematical model $\mathbf{m}$.

In solving real task of controlling the CTP, a situation may occur when a unified model $\mathbf{m}$

In this case, you can use the following. The region $U$ is divided into subsets $U_{i}, i=\overline{1, n}$, such that

$U=\bigcup_{i=1, n} U_{i}$ и $\forall(i \neq j) U_{i} \bigcap U_{j}=\varnothing ; i, j=\overline{1, n}$.

Then, for each $U_{i}$, an auxiliary model $\mathbf{m}_{i}$ is selected that satisfies the conditions of the theorems.

The set of models $\left\{\mathbf{m}_{i}\right\}$ can be used as an auxiliary model for solving task two-model optimization. We will call such a model a generalized auxiliary model and denote, as before, $\mathbf{m}$. Thus, in this case $\mathbf{m}=\left\{\mathbf{m}_{i}\right\}$.

However, it is necessary that the mathematical models satisfy the conditions of the theorems.

We will call the conditions of the theorems the conditions of suitability of the operators $\mathbf{m}$ and $\boldsymbol{M}$.

If the conditions of the theorems are not satisfied, then the auxiliary model $\mathbf{m}$ should be replaced by another more complex model or a generalized model $\left\{\mathbf{m}_{i}\right\}$, for which the conditions of the

If the auxiliary model cannot be found, then it is necessary to investigate the possibility of using the simulation model 
$\boldsymbol{M}(\overline{\mathbf{x}}, \mathbf{u}, \overline{\mathrm{b}})$ to solve the problem of guaranteeing optimization (where $\overline{\mathrm{x}}, \overline{\mathrm{b}}$ - input magnitude fashion).

Model $\boldsymbol{M}$ can be used when fitness conditions are met. Otherwise, there remains only the opportunity to solve the problem of guaranteeing optimization by direct non-linear programming methods.

The algorithm for choosing a method for solving the problem of guaranteeing optimization under conditions of uncertainty is as follows.

1. The auxiliary model $\mathbf{m}$ is synthesized, including the system of auxiliary models $\left\{\mathbf{m}_{\mathrm{i}}\right\}$ (that is, the generalized auxiliary model).

2. If a model $\mathrm{m}$ is found, then it is possible to use the $\alpha$ optimization method with an auxiliary model m (two-model optimization method).

3. If a generalized model $\left\{\mathbf{m}_{\mathrm{i}}\right\}$ is not found, then the conditions of suitability of the model $\boldsymbol{M}$ are checked.

4. If the conditions for the suitability of the model $\boldsymbol{M}$ are satisfied, then the $\alpha$-optimization method with the simulation model $\boldsymbol{M}$ can be applied. Otherwise, they proceed to the solution of the problem by the direct method.

Let us describe the block diagram of the algorithm for the synthesis of a generalized auxiliary model, i.e. item 1 of the algorithm for choosing a solution method.

In block 1 in quality multitude manager $\bar{U}$ influence assigned to multitude $U$.

In block 2 , the auxiliary model $\mathbf{m}$, which is designed to work on the whole set, is selected $\bar{U}$ and sequentially complicated.

In block 3, the suitability conditions for model $\mathrm{m}$ are checked. If the suitability conditions are satisfied, then the problem of guaranteeing optimization is solved by the method of two-model optimization with the found model m (block 4).

Otherwise, in block 5, the question of the advisability of complicating the mathematical model $\mathbf{m}$ is solved.

If the search possibilities for the model $\mathbf{m}$ suitable for the entire area $\bar{U}$ are exhausted, then control is transferred to block 6 .

In block 6 , the results of testing the suitability conditions performed in block 3 are analyzed, and based on this analysis, the area managers (subsets) multitude manager $U_{\mathrm{i}}$ for which the suitability conditions are satisfied.

If this area is empty (block 7), then this means that the auxiliary model $\mathbf{m}$ could not be found. In this case, control is transferred to block 8 .

Otherwise, this subset is remembered and at the same time the corresponding mi model $\mathbf{m}_{\mathrm{i}}$ found for it is remembered.

In block 9, the multitude $\bar{U}$ is formed, which is equal to the difference of the previous set and the selected subset $U_{\mathrm{i}}$. Then, in block 2 , the selection and subsequent complication of the model $\mathrm{m}$ begins on the new multitude $\bar{U}$.

This iterative process ends either when, at the next iteration, the subset $U_{\mathrm{i}}$ is empty (block 7), or when the validation of the suitability conditions is successful (block 3 ).

In the latter case, the auxiliary model $\mathbf{m}$ is synthesized

As follows from the synthesis algorithm, the model $\mathbf{m}$ can be both the only one acting on the whole set of control actions $\bar{U}$ and the generalized model $\mathbf{m}=\left\{\mathbf{m}_{\mathrm{i}}\right\}$, that is, set of mi models $\mathbf{m}_{\mathrm{i}}$, each of which acts on the corresponding subset $U_{\mathrm{i}}$.

If the next subset $U_{\mathrm{i}}$ is empty and the generalized model $\left\{\mathbf{m}_{\mathrm{i}}\right\}$ is not found, then in blocks 8 and 10, the fulfillment of the conditions for the suitability of the simulation model $\boldsymbol{M}$ is checked.
If the suitability conditions are fulfilled, then the solution of the guaranteeing optimization problem under uncertainty is performed by the $\alpha$-optimization method with the simulation model $\boldsymbol{M}$. Otherwise, the initial problem is solved by direct methods in block 12 .

The choice and subsequent complication of the auxiliary model $\mathbf{m}$, carried out in block 2 , is a complex task that must be solved interactively using mathematical methods of sequential complication of the model.

Such methods are the modified Chebyshev method of subsequent complications with the estimation of residual variance $[11,12]$, used to construct algebraic models, the use of aggregation methods [13] or reduction [14] for differential operators, and the use of integral operators with increasing complexity for the integral type of the operator $\mathbf{m}$.

The choice of operator type (algebraic, differential, integral) is carried out by the decision maker in an interactive mode.

In the interactive mode, a decision is made on the correction of mathematical models, as well as on the use of mixed types of operators.

To test the effectiveness of the proposed method, let us consider the problem of guaranteed optimization of the process of obtaining Scheffer's acid in a microwave reactor, which is formulated as: for a given particle size distribution of 2-naphthol $\phi(\mathrm{r})$ entering into a chemical reaction, it is necessary to find the consumption $\mathrm{G}_{\mathrm{s}}$ and concentration $\mathrm{C}_{0}$ sulfuric acid at the entrance to the microwave reactor, at which the degree of conversion of 2-naphthol into Scheffer's acid $C$ reaches its maximum value, i.e.

$Q\left(u^{*}\right)=\max _{u \in U} Q(u)$

where $Q(u)=\min C \mid \mu_{\tilde{K}}(C \mid \mathrm{u}) \geq \mu_{3}, \quad \mu_{3}$ - the set value of the membership function of the degree of conversion of 2naphthol into Schaeffer's acid; when satisfying the relationships determined by the mathematical model [4]; with guaranteed fulfillment of technological requirements

$\sigma \leq \underline{\sigma}^{b}$, where $\underline{\sigma}^{b}-$ border of significance of the content of by-products and sulfones in the finished product, determined by the formula

$$
\underline{\sigma}^{b}=\min _{E_{b}} \sigma
$$

where $E_{\sigma}=\left\{\sigma \mid \mu(\sigma \mid \mathrm{u}) \geq \varepsilon_{\sigma}-\right.$ significance region; $\varepsilon_{\sigma}-$ significance level the content of by-products and sulfones in the finished product.

Region control actions $\mathrm{U}$ determined by the following restrictions:

$\mathbf{C}_{0}^{\min } \leq \mathbf{C}_{0} \leq \mathbf{C}_{0}^{\max } ; \quad G_{s}^{\min } \leq G_{s} \leq G_{s}^{\max }$

Below is an analysis of the influence of the particle size distribution of 2-naphthol $\phi(\mathbf{r})$ on optimal control actions $G_{\mathrm{s}}$ and $C_{0}$ and values of the degree of conversion of 2naphthol into Schaeffer's acid. As an example Fig. 1 shows the results of solving the optimization problem.

Analysis of the calculated data shows that the optimal values of control actions significantly depend on the average radius of 2-naphthol particles. With an increase in the consumption of sulfuric acid, the temperature of the reaction mixture, the residence time of the reaction mixture in the 
reactor, and the amount of 2-naphthol disulfonic acids decrease, which leads to an increase in the conversion of 2naphthol into Scheffer's acid for 2-naphthol particles with an average size of $5 \ldots 7 \mu \mathrm{m}$. An increase in the concentration of sulfuric acid leads to a more rapid dissolution of 2-naphthol particles, however, the formation of 2-naphthol disulfonic acids, which are by-products, significantly increases. As a result, the degree of conversion of 2-naphthol to Scheffer's acid is reduced.

$G_{s}^{*}, \mathrm{~kg} / \mathrm{h}$

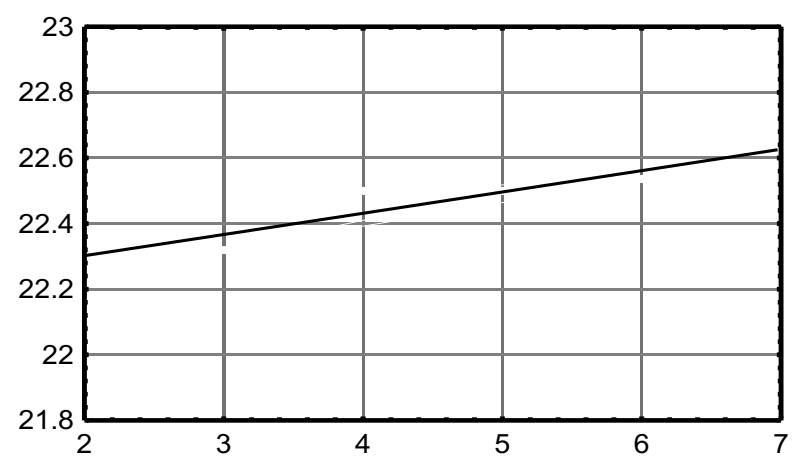

$\mathrm{r}_{0}, \mu \mathrm{m}$

Fig. 1. Dependence of the optimal consumption of sulfuric acid $G_{s}^{*}$ from the average particle size of 2-naphthol

Experimental studies of the optimal regimes for the synthesis of Scheffer's acid in a microwave reactor were carried out. A fragment of the research results are shown in Table 1.

Analysis of the data in Table 1 shows that the degree of conversion of 2-naphthol to Scheffer's acid under optimal conditions of sulfonation of 2-naphthol to Scheffer's acid ranges from $81 \ldots 88 \%$, while the formation of resins and sulfones not exceed $0.3 \%$, and synthesis time no more than 10 min. Good agreement between the calculated and experimental data confirms the reliability of the results obtained.
Table 1. Experimental data on the synthesis of Scheffer's acid

\begin{tabular}{l|l|l|l|l}
\hline \multicolumn{2}{l|}{ Control actions } & & \multicolumn{2}{l}{ Parameter values } \\
\hline $\mathrm{G}_{\mathrm{s}}, \mathrm{kg} / \mathrm{h}$ & $\mathbf{C}_{\mathbf{0}}, \mathbf{\%}$ & $\mathbf{r}_{\mathbf{0}}, \boldsymbol{\mu m}$ & $\mathbf{C} \boldsymbol{,} \%$ & $\boldsymbol{\sigma}, \mathbf{\%}$ \\
\hline 22,10 & 83,5 & 2,8 & 87 & 0,19 \\
22,10 & 84,4 & 4,2 & 84 & 0,25 \\
22,10 & 83,5 & 6,7 & 81 & 0,32 \\
22,15 & 83,5 & 2,8 & 88 & 0,21 \\
22,45 & 84,4 & 4,2 & 86 & 0,23 \\
22,45 & 84,4 & 6,7 & 83 & 0,29 \\
22,80 & 83,5 & 2,8 & 82 & 0,28 \\
22,80 & 85,8 & 4,2 & 84 & 0,23 \\
\hline
\end{tabular}

Thus, the optimization of the static regimes of the microwave sulfonation reactor makes it possible to increase the degree of conversion of 2-naphthol into Scheffer's acid and to reduce the content of resins and sulfones in the finished product.

\section{Conclusion}

A method is proposed for solving problems guaranteeing the optimization of chemical-tech-nological processes under conditions of uncertainty, which can significantly reduce the time to solve such problems. Algorithms for the synthesis of an auxiliary mathematical model and the choice of a method for solving the problem of guaranteeing optimization under uncertainty are given. Using the $\alpha$-optimization method with a simulation model reduces the time required to solve the problem of guaranteeing optimization under conditions of uncertainty by an order of magnitude, and the two-model optimization method by two or more orders of magnitude.

This is an Open Access article distributed under the terms of the Creative Commons Attribution License

\section{References}

1. V.I. Bodrov, Yu.Yu. Gromov, V.A. Pogonin "Decision tasks guaranteeing optimization in conditions uncertainties", Engineering Physics, No.2, pp.46-48 (2003).

2. V.V. Kafarov, V.I. Bodrov, V.G. Matveykin "Problems of managing deterministic stochastic models", Reports of the USSR Academy of Sciences, Vol.308, No.3, pp.663-666 (1989).

3. Sanders, J. Stochastic optimization of large-scale complex systems. Technische Universiteit Eindhoven. 247 (2016).

4. G. Ostrovsky,T. Lapteva, N. Ziyatdinov "Optimal design of chemical processes under uncertainty", Theoretical Foundations of Chemical Engineering, No.48,pp. 583-593 (2014). doi:10.1134/ S0040579514050212.

5. T. Lapteva, G. M. Ostrovsky, N. N. Ziyatdinov "Optimization of chemical technology processes under probabilistic constraints",Theoretical Foundations of Chemical Engineer$\begin{array}{llll}\text { ing, 44(5), } & \text { pp. } & 651 & -659 \text { (2010). }\end{array}$ DOI: $10.1134 / \mathrm{S} 0040579510050039$.

6. V.V. Kafarov, V.I. Bodrov, V.G. Matveykin "Theoretical provisions for solving deterministic-stochastic models control problems", Reports of the USSR Academy of Sciences, Vol.317, No.4, pp.927-931(1991).

7. Erschen S. Duddeck F. Gerdts M. Zimmermann M. "On the Optimal Decomposition of High-Dimensional Solution Spaces of Complex Systems". ASCE-ASME Jornal of Rick and Uncertainty in Engineering System. Vol.4.Issue 2 (2018). doi.org/10.1115/1.4037485.
8. N. N. Ziyatdinov "Modeling and Optimization of Chemical Engineering Processes and Systems", Theoretical Foundations of Chemical Engineering, 51(6), pp. 889-892 (2017) doi: 10.1134/ S0040579517060197

9. Biegler, L.T. "Integrated Optimization Strategies for Dynamic Process Operations". Theor Found Chem Eng 51, 910-927 (2017). doi.org/10.1134/S004057951706001X.

10. A. Wechsung, J. Oldenburg, J. Yu, A. Polt "Supporting chemical process design under uncertainty" Brazilian Journal of Chemical Engineering, 27(3) with 189 Reads (2010). doi:10.1590/ S010466322010000300009.

11. Notaris S.E."Product integration rules for Chebyshev weight functions with Chebyshev abscissae". Journal of Computational and Applied MathematicsFebruary. (2014). doi.org/10.1016/j.cam. 2013.08.013

12. Xingxing Lv. Shen S. "On Chebyshev polynomials and their applications".(2017). doi: 10.1186/s13662-017-1387-8

13. Sun Y. Halgamuge S.K. Kirley M. "On the Selection of Decomposition Methods for Large Scale Fully Non-separable Problems" (2015). doi:10.1145/2739482.2768483.

14. Iqbal J. Ullah M. Khan S. G. Khelifa B. and Ćuković S."Nonlinear control systems - A brief overview of historical and recent advances". Nonlinear Engineering .Vol. 6: Issue 4 (2017). doi.org/10.1515/nleng-2016-0077 . 